\title{
Thermodynamic analysis and energy efficiency of thermal desalination processes
}

\author{
Doriano Brogioli ${ }^{\mathrm{a}, *}$, Fabio La Mantia ${ }^{\mathrm{a}}$, Ngai Yin Yip ${ }^{\mathrm{b}}$ \\ ${ }^{\text {a } U n i v e r s i t a ̈ t ~ B r e m e n, ~ E n e r g i e s p e i c h e r-~ u n d ~ E n e r g i e w a n d l e r s y s t e m e, ~ B i b l i o t h e k s t r a ß e ~ 1, ~ B r e m e n ~ 28359, ~ G e r m a n y ~}$ \\ b Department of Earth and Environmental Engineering and Columbia Water Center, Columbia University, New York, NY 10027-6623, United States
}

\section{A R T I C L E I N F O}

\section{Keywords:}

Desalination

Thermal separation

Physical limitations

Salinity gradient power

Vacuum distillation

Membrane distillation

\begin{abstract}
A B S T R A C T
This thermodynamic study examines the principles governing energy efficiency and specific energy requirement intrinsic to thermal desalination processes. The practical performances of desalination technologies are investigated and related to the fundamental physical limitations of the processes. The energy efficiency of any thermal desalination process fulfils a limitation similar to the well-known Carnot law for heat engines. The efficiency of a single-effect distiller is limited by a function of the boiling point elevation of the solution. Further analysis shows that, although this can appear as paradoxical, a higher energy efficiency is obtained by a solution with higher boiling point elevation. For multiple-effect distillation, the limit also depends on the temperature drops across the heat exchangers. Comparison with empirical data indicates that these limits are actually approached in real plants. Our study discusses the thermodynamic framework for understanding the performances of thermally-driven desalination technologies, emphasizing the role of the boiling point elevation. The results can be also seen as rules-of-thumb for designing and evaluating the performances of a multiple-effect distillation unit, based only on the properties of the solution to be distilled, in particular, the boiling point elevation.
\end{abstract}

\section{Introduction}

Addressing our global water challenge is one of the most urgent priorities for the 21st century [1-4]. Water demand is projected to increase by $\approx 55 \%$ in 2050 due to increase in population and living standards [5]. While seawater desalination can expand our water supply $[6,7]$, the process is energy intensive and costly and, hence, is usually considered the "last resort" option [7-9]. To improve the sustainability of desalination, it is imperative to pursue further fundamental research on the desalination technologies.

Thermally-driven multiple-effect distillation (MED) and multi-stage flash (MSF), and membrane-based reverse osmosis and electrodialysis are the leading desalination technologies [7-10]. Studies have examined the thermodynamic efficiency of practical reverse osmosis and electrodialysis processes, and determined the specific energy requirement to desalinate seawater [11-13]. Similar investigations looked at MED and MSF [14-23]. The efficiencies, energy consumption, and gained output ratios determined from these foundational studies are in agreement with empirical data of actual thermal desalination facilities and the findings of these studies advanced the understanding of thermal desalination processes. However, the role played by thermo-physical parameters of the solution, such as boiling point elevation, are not specifically examined, and the relationship with energy efficiency is not elucidated. Just as the Carnot efficiency, derived from the first principles of thermodynamics, is the theoretical upper limit for heat engines, the second law of thermodynamics is also intuitively expected to influence the limits of separation efficiency for thermally-driven processes. Therefore, the efficiency and energy requirement of thermal desalination processes, i.e., MED and MSF, are fundamentally related to the temperatures of the heat source and heat sink, and thermodynamic pathways.

This study presents an analytical approach to explicitly discuss the effect of some of the thermo-physical parameters (in particular, the boiling point elevation) and to unveil their qualitative role. This approach complements existing studies and provides an alternate perspective on the principles governing separation by distillation. Specifically, we describe a thermodynamic framework for understanding the principles governing energy efficiency and specific energy requirement intrinsic to thermal desalination processes. Firstly, the basis of a Carnot-like efficiency for a thermodynamically reversible thermal separation process is introduced. The theoretical efficiency of hypothetically distilling an infinitesimally small volume of water from a saline feed is then examined. The analysis is then extended to evaluate the efficiency of a real batch process taking place in a single-effect

\footnotetext{
"Corresponding author.

E-mail addresses: brogioli@uni-bremen.de (D. Brogioli), lamantia@uni-bremen.de (F. La Mantia), n.y.yip@columbia.edu (N.Y. Yip).
} 
distiller, and compared with the above-mentioned thermodynamic limit. Next, we describe application of the approach to a multiple-effect distillation unit with heat exchangers, to model performance of actual desalination in practical thermal distillation unit operation. Lastly, the efficiency and energy requirement of thermal distillation is compared with membrane-based technologies of reverse and electrodialysis. The results of this thermodynamic study emphasize the importance of the fundamental governing equations that define the limits of thermal desalination efficiency, clarifying the role of some thermo-physical parameters and in particular of the boiling point elevation. Our analysis can be a rule-of-thumb approach for designing and evaluating the performance of a multiple-effect distillation unit.

\section{Expression of the Carnot law for separation processes}

The analysis of distillation processes for desalination in the context of the second law of thermodynamics has been successfully employed to identify the sources of entropy generation, with the purpose of minimizing the losses [19-22]. This analysis led to the definition of the energy efficiency and second-law efficiency of the process, together with the minimum least work of separation. Analogous definitions of efficiencies have been given for distillation in liquid mixtures [24]. Here we report a synthetic derivation of the efficiencies for the specific scope of the present paper; a more detailed discussion can be found in literature $[19,20,20,22]$.

The solution to be distilled, the brine and the pure solvent are all at temperature $T_{\mathrm{L}}$. The distillation is driven by a heat source at temperature $T_{\mathrm{H}}$. We define the efficiency $\eta$ of the distillation as:

$\eta=\frac{\Delta G}{Q_{\mathrm{H}}}$,

where $Q_{H}$ is the heat adsorbed from the heat source and $\Delta G$ is the increase of Gibbs free energy from the feed solution to the concentrated solution and pure solvent, evaluated at $T_{\mathrm{L}}$. In literature, the quantity $\Delta G$ is often expressed as the least work of separation or as the minimum least work of separation, see Eqs. (4) and (6) of Ref. [19]; similar concepts are used in separation of any chemicals [25].

From the principles of thermodynamics, we can derive an expression for the maximum efficiency of the distillation, equivalent to the Carnot law.

From the first principle:

$Q_{\mathrm{H}}-Q_{\mathrm{L}}=\Delta H$,

where $\Delta H$ is the variation of enthalpy of the solutions induced by the distillation and $Q_{\mathrm{L}}$ is the heat released to the heat sink.

The second principle states that:

$\Delta S+\Delta S_{\mathrm{e}} \geq 0$,

where $\Delta S$ is the variation of entropy of the solutions induced by the distillation and $\Delta S_{\mathrm{e}}$ is the variation of the entropy of the environment. This leads to:

$-\frac{Q_{\mathrm{H}}}{T_{\mathrm{H}}}+\frac{Q_{\mathrm{L}}}{T_{\mathrm{L}}}>-\Delta S$.

By combining Eqs. (2) and (4), we obtain:

$\left(1-\frac{T_{\mathrm{L}}}{T_{\mathrm{H}}}\right) Q_{\mathrm{H}}>\Delta H-T_{\mathrm{L}} \Delta S$.

From the definition of $\Delta G=\Delta H-T \Delta S$, at $T=T_{\mathrm{L}}$, we obtain:

$\left(1-\frac{T_{\mathrm{L}}}{T_{\mathrm{H}}}\right) Q_{\mathrm{H}}>\Delta G$

From the definition of $\eta$, Eq. (1):

$\eta<1-\frac{T_{\mathrm{L}}}{T_{\mathrm{H}}}$.
It is to be noticed that here $T_{\mathrm{H}}$ and $T_{\mathrm{L}}$ are the temperatures of the heat source and sink. In literature, this law is often expressed in terms of the least heat of separation, see Eq. (9) of Ref. [19].

Now we discuss the "second-law efficiency" $\eta_{2 \text { nd-law }}$, or exergy efficiency [22]. It is connected to the entropy production $\Delta S+\Delta S_{\mathrm{e}}$ taking place when an amount of heat $Q_{\mathrm{H}}$ is adsorbed by the process from the heat source. First we define the "entropy production efficiency" $\sigma$ as the ratio between the entropy production $\Delta S+\Delta S_{\mathrm{e}}$ and the maximum possible entropy production which would take place by simple heat conduction of $Q_{\mathrm{H}}$ from the heat source to the heat sink:

$\sigma=\frac{\Delta S+\Delta S_{\mathrm{e}}}{\frac{Q_{\mathrm{H}}}{T_{\mathrm{L}}}-\frac{Q_{\mathrm{H}}}{T_{\mathrm{H}}}}$.

During distillation, the feed is divided into two different solutions with a concentration difference, hence there is an entropy decrease: $\Delta S<0$. We thus see that $\sigma$ is bound between 0 (the total entropy must increase; the limit is reached when $\Delta S=-\Delta S_{\mathrm{e}}$ ) and 1 (heat $Q_{\mathrm{H}}$ freely flows from the heat source to the heat sink, thus $\Delta S=0$ and $\Delta S_{\mathrm{e}}=Q_{\mathrm{H}}$ $\left(1 / T_{\mathrm{L}}-1 / T_{\mathrm{H}}\right)$.

We define the "second-law efficiency" $\eta_{2 \text { nd-law }}$ as the complementary fraction of the entropy production efficiency $\sigma$ :

$\eta_{2 \text { nd-law }}=1-\sigma=1-\frac{\Delta S+\Delta S_{\mathrm{e}}}{\frac{Q_{\mathrm{H}}}{T_{\mathrm{L}}}-\frac{Q_{\mathrm{H}}}{T_{\mathrm{H}}}}$

which is 1 when no entropy is produced, i.e. the equality holds in Eq. (3), and is 0 when no distilled solution is produced, but rather all the heat $Q_{\mathrm{H}}$ is transferred to the heat sink at $T_{\mathrm{L}}$.

We can write the second-law efficiency $\eta_{\text {2nd-law }}$ in terms of the efficiency $\eta$. First we write the variation of entropy of the environment $\Delta S_{\mathrm{e}}$ as:

$\Delta S_{\mathrm{e}}=\frac{Q_{\mathrm{L}}}{T_{\mathrm{L}}}-\frac{Q_{\mathrm{H}}}{T_{\mathrm{H}}}$.

Then we write the variation of entropy of the system $\Delta S$ in terms of the free energy variation $\Delta G$ :

$\Delta S=\frac{-\Delta G+\Delta H}{T_{\mathrm{L}}}$

we express $\Delta G$ in terms of $\eta$ from Eq. (1) and $\Delta H$ in terms of heats $Q_{H}$ and $Q_{\mathrm{L}}$ from Eq. (2):

$\Delta S=\frac{-\eta Q_{\mathrm{H}}+Q_{\mathrm{H}}-Q_{\mathrm{L}}}{T_{\mathrm{L}}}$.

We finally get:

$\eta_{\text {2nd-law }}=\frac{\eta}{\left(1-\frac{T_{\mathrm{L}}}{T_{\mathrm{H}}}\right)}$,

i.e. $\eta_{2 \text { nd-law }}$ represents the ratio between the actual energy efficiency $\eta$ and the efficiency of an ideal Carnot cycle; Eq. (7) is equivalent to $\eta_{2 n d-}$ law $\leq 1$. Analogous expressions have been reported in literature, see Eq. (17) of Ref. [19], Eq. (72) of Ref. [20], Eq. (4.138) of Ref. [22].

\subsection{Alternative derivation}

This law can be obtained from a different point of view; an analogous derivation for distillations of liquid mixtures can be found in Ref. [24]. Consider a black box process that has heat input at $T_{\mathrm{H}}$, and heat output at $T_{\mathrm{L}}$ together with work production (see Fig. 1).

In the process on the left, the black box process is the classic Carnot cycle:

$\eta_{\text {work }}=\frac{W}{Q_{\mathrm{H}}}=1-\frac{T_{\mathrm{L}}}{T_{\mathrm{H}}}$.

In the process on the right, the black box consist of two separate processes. The 1st is a thermal separation of a saline feed into a high 




Fig. 1. Distillation process (left) and distillation process coupled to a salinity-gradientpower device (right).

and low concentration solutions. We define the efficiency of thermal separation:

$\eta_{\text {sep }}=\frac{\Delta G_{\text {sep }}}{Q_{\mathrm{H}}}$.

The so-called "salinity gradient power" devices are able to produce work by the controlled mixing of solutions at different concentrations (examples are the reverse electrodialysis [26,27] (RED) and the pressure-retarded osmosis [28-30]) (PRO). In principle, under reversible thermodynamic conditions, such devices can produce a work $W=\Delta G$. We thus see that Eq. (7) corresponds to the usual Carnot law by substituting $\Delta G$ with $W$.

Now we consider that the two solutions (produced by distillation) are mixed in an ideal PRO or RED process to produce work. We have already shown that in thermodynamically reversible PRO and RED processes [31,32], the work produced is exactly equal to the Gibbs free energy of mixing:

$W=-\Delta G_{\text {mix }}$.

Because the Gibbs free energy of mixing is equal and opposite to the Gibbs free energy of separation, and the efficiency of work production is the same despite the different paths in the left and right black boxes,

$\eta_{\text {sep }}=\frac{\Delta G_{\text {sep }}}{Q_{\mathrm{H}}}=\frac{-\Delta G_{\text {mix }}}{Q_{\mathrm{H}}}=\frac{W}{Q_{\mathrm{H}}}=\eta=1-\frac{T_{\mathrm{L}}}{T_{\mathrm{H}}}$.

We arrive at the same maximum efficiency for thermal separation processes, Eq. (7).

\section{Distillation technologies}

Facility-scale thermal distillation of saline feed water is performed by various technologies, mainly i) multiple-effect distillation (MED), ii) multi-stage flash (MSF), or iii) mechanical vapor compression (MVC) [33,34].

In the present paper, we neglect MVC because it involves mechanical work and not only heat exchanges, as required by the hypothesis we used for obtaining Eq. (7).

We focus on MED, which is composed by multiple "effects" (stages) in series. An example of realization of each effect is shown in Fig. 2. The drawing is aimed at facilitating the explanation of the concepts and it is not intended to actually represent a real thermal desalination process. The "effect" is composed by two parts, the evaporation and condensation compartments. In the evaporation compartment, the solution to be distilled is heated by the heat source until the solution boils. The steam is then cooled down in the condensation unit, by dissipating heat to the heat sink, until it condenses. Air and other gasses are removed from the vessels (hence the name "vacuum distillation"), so that the pressure $P$ inside the effect determined by the gas-liquid equilibria of the solution and of the pure solvent at the temperatures of the chamber. It is important to notice that no mechanical work is needed in principle to maintain the vacuum; the injection of further solution to be distilled and the extraction of the concentrated solution can be performed without a significant mechanical work, thanks to the substantial incompressibility of the liquids. In practice, the presence of dissolved air in the feed solution leads to the release of gasses in the chambers, that must be continuously removed by pumps; however, the power consumption for this process is usually negligible.

In MEDs, each effect (stage) of the device operates at a different pressure $P$; for this reason, we will consider $P$ as a parameter. Usually $P$ is lower than $1 \mathrm{~atm}$; however, we will consider $P=1 \mathrm{~atm}$ when possible in order to have a simple comparison with a usual situation.

The calculations we will show hold also in the case of the so-called "membrane distillation", provided that $P$ is considered as the partial pressure of the solvent vapor.

In Fig. 3, we show three effects connected in series, forming a MED unit. The heat released by condensation is used for evaporating the solvent of the subsequent effect.

The following discussion could be extended to MSF; however, it is a widely held opinion that MSF has a lower thermodynamic efficiency than MED, due to the inherently out-of-equilibrium operation.

\section{Single effect distillation}

In this section, we consider a single effect, i.e. a single stage of a MED device. The "effect" is modeled with the following assumptions:

- The pressure $P$ is uniform in the effect.

- No mechanical work is performed by (nor on) the vapor for changing its volume or pressure.

\subsection{Carnot law for single-effect distillation}

First we derive a general law for the maximum efficiency of each effect of a MED, under the general hypothesis mentioned above, which hold in real thermal desalination process based on MED. A similar analysis for mixtures of two liquids can be found in Ref. [24]. We do not rely on a specific description of the detail of the process, but rather we base our calculation on general hypothesis which hold for real devices. It is worth noting that the maximum efficiency we evaluate cannot be reached in real devices; however, it can be roughly approached, as will be discussed later in the paper.

We call $T_{\mathrm{bp}}(P, x)$ the boiling point of the solution at pressure $P$ and molar fraction $x$. In the case of a single pressure $P$ in the device, we will drop the first variable $P$ and write $T_{\mathrm{bp}}(x)$. We will consider isobaric processes, hence the variation of enthalpy equals the exchanged heat.

We call $x_{0}$ the concentration of the brine, the by-product of the production of the pure solvent. For the purpose of explaining the concept, we can imagine infinitely large hypothetical chambers, with an infinitely large volume of feed and also infinitely large headspace, such that the boiling-off of a drop of water will not change the concentration of the saline feed, nor will the pressure of the headspace be affected. Likewise for the condensation chamber. Under such simplified view, $x_{0}$ also equals the concentration of the feed solution. In real devices, the concentration of the solution increases during boiling; in the following, which is of general validity, $x_{0}$ will represent the maximum molar fraction of the solution inside the effect.

During the operation of the effect, when a given amount of separation work, $\Delta G$, is performed, an amount of heat $Q_{H}$ must be provided to the fluids. In principle, the process can take place by using a heat source at a temperature $T_{\mathrm{H}}$ equal to $T_{\mathrm{bp}}\left(x_{0}\right)$ and a heat sink at a temperature $T_{\mathrm{L}}$ equal to $T_{\mathrm{bp}}(0)$ (the two temperatures represent the maximum and minimum temperatures of the fluids respectively); from Eq. (7), we obtain:

$\eta_{\text {single-effect }}=\frac{\Delta G}{Q_{\mathrm{H}}} \leq 1-\frac{T_{\mathrm{bp}}(0)}{T_{\mathrm{bp}}\left(x_{0}\right)}=\eta_{\text {single-effect }}^{\max }$.

Although the use of a heat source at $T_{\mathrm{H}} \rightarrow T_{\mathrm{bp}}\left(x_{0}\right)$ is not practical, 


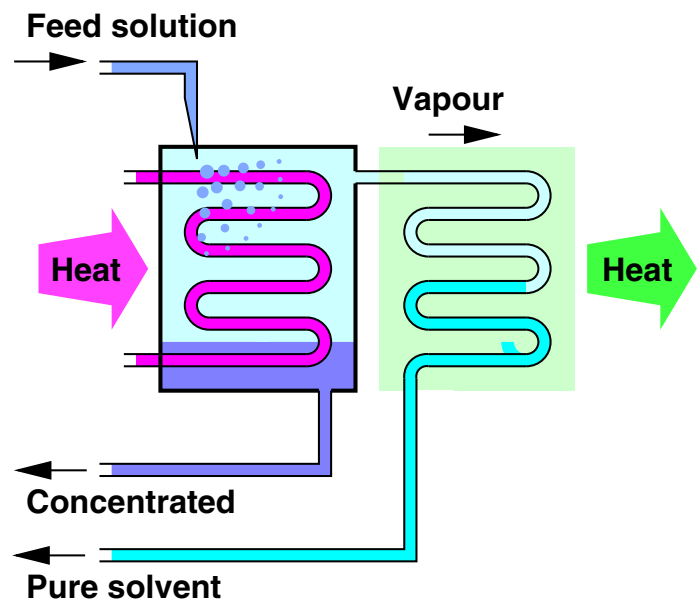

Fig. 2. Scheme of a single effect distiller.

because it would lead to a vanishing heat exchange, it allows us to obtain a relation, similar to the Carnot law, which only involves physical properties of the solutions: $\Delta G, Q_{\mathrm{H}}, T_{\mathrm{bp}}\left(x_{0}\right)$ and $T_{\mathrm{bp}}(0)$.

On the other hand, the use of $T_{\mathrm{H}}$ higher than $T_{\mathrm{bp}}\left(x_{0}\right)$ and $T_{\mathrm{L}}$ lower than $T_{\mathrm{bp}}(0)$, by a given temperature difference $\delta T$, increases the speed of the process to a finite value, thanks to a larger temperature gradient across the heat exchangers. However it does not improve the efficiency; actually, the entropy production of an actual process is larger due to the passive conduction of heat across the heat exchangers. Indeed, supplying heat to the solution to bring it up from $T_{\mathrm{L}}$ to $T_{\mathrm{bp}}(0)$ does not perform any separation - we are just merely heating up the solution. Likewise, increasing the steam temperature from $T_{\mathrm{bp}}\left(x_{0}\right)$ to $T_{\mathrm{H}}$ is also not carrying out any separation, just superheating the steam. The effective temperatures where separation occurs is $T_{\mathrm{bp}}(0)$ and $T_{\mathrm{bp}}\left(x_{0}\right)$.

From Eq. (18), we thus see that the efficiency $\eta$ does not depend on the realization of the distillation unit, nor on the temperature of the available heat source. One of the consequences is that the efficiency of the whole device cannot be increased by supplying heat at a temperature higher than $T_{\mathrm{bp}}\left(x_{0}\right)$.

The boiling temperatures that appear in Eq. (18) depend on the pressure $P$, hence the maximum efficiency $\eta_{\text {single-effect }}^{\max }$ should also depend on $P$. We can approximately evaluate the variation of $\eta_{\text {single-effect }}^{\max }$ with the pressure $P$ by using the "Dühring's rule" [35], which gives an approximated evaluation of the boiling temperature as a function of the pressures $P$, at least approximately, from the boiling temperatures $T_{\mathrm{bp}}$ $\left(P_{0}, x_{0}\right)$ and $T_{\mathrm{bp}}\left(P_{0}, 0\right)$ at a given pressure $P_{0}$. Dühring's rule states that an approximately linear relationship exists between the temperatures at which two solutions exert the same vapor pressure. A liquid boils when its vapor pressure equals the pressure of its environment, so we can rephrase the Dühring's rule by saying that there is a direct proportionality

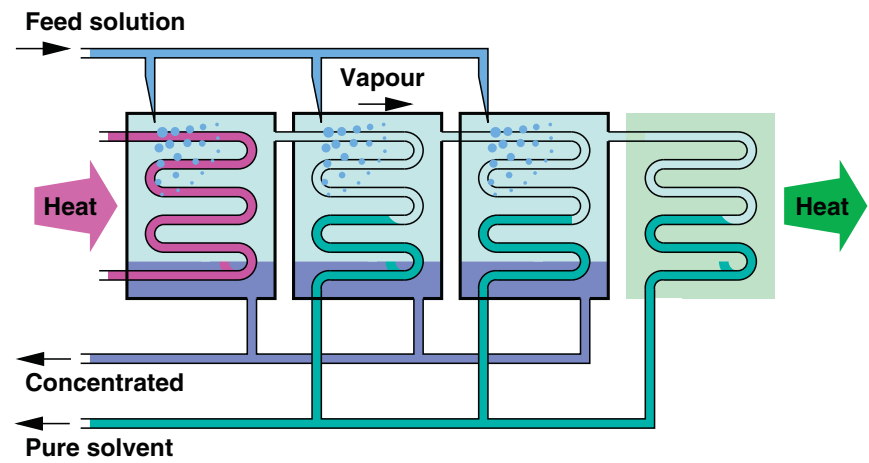

between the boiling temperatures at two different concentrations $x_{1}$ and $x_{2}$, as a function of the pressure in the effect:

$\frac{T_{\mathrm{bp}}\left(P, x_{1}\right)}{T_{\mathrm{bp}}\left(P, x_{2}\right)}=\frac{T_{\mathrm{bp}}\left(P_{0}, x_{1}\right)}{T_{\mathrm{bp}}\left(P_{0}, x_{2}\right)}$.

This shows that $T_{\mathrm{bp}}\left(P, x_{1}\right) / T_{\mathrm{bp}}\left(P, x_{2}\right)$ is approximately independent on $P$, for any couple of values of $x_{1}$ and $x_{2}$. This leads to the conclusion that $\eta_{\text {single-effect }}^{\max }$ is approximately a constant, i.e. it is approximately independent on the pressure and, in turn, on the condensation temperature, which finally defines the pressure in the effect after evacuation of air.

In the following, we will use the "relative efficiency" $\epsilon_{\text {single-effect, }}$ that we define as:

$\epsilon_{\text {single-effect }}=\frac{\eta_{\text {single-effect }}}{\eta_{\text {single-effect }}^{\text {max }}}$

It can be easily seen that this definition allows us to rewrite Eq. (18) as $\epsilon_{\text {single-effect }} \leq 1$. This last equation bears analogies with Eq. (13), although $\epsilon_{\text {single-effect }}$ does not rigorously represent a second-law efficiency.

It has been shown [36] that $\eta_{\text {single-effect }}$ can roughly approach its maximum value $\eta_{\text {single-effect }}^{\max }$ (see Eq. (18)) in conditions that can be realized in the laboratory; in other words, the relative efficiency $\epsilon_{\text {single-effect }}$ can roughly approach 1 ; actually, also real industrial processes can reach a relevant percentage. In order to discuss this possibility, in the next sections we will model various distillation processes.

\subsection{Batch process description and model}

In this section, we discuss a hypothetical "thought" experiment, aimed at facilitating the explanation of the concepts; it is not intended to actually represent a real thermal desalination process. It is a simplified version of a single stage of the above-mentioned MED. We use additional assumptions, in particular, we assume that the insulation is perfect (no heat is lost to the surrounding). However, we include some relevant phenomena representing the real distillation process, in particular, we account for the increase of concentration during the vaporization of the solvent.

The first simplified batch process that we consider is shown in Fig. 4. The whole process takes place at a constant pressure $P$; it starts with a solution $S_{1}$ at concentration $x_{1}$ and temperature $T_{\mathrm{bp}}(x=0)$, and is composed of the following four phases:

1. The total amount of solution $S_{1}$ at molar fraction $x_{1}$ is heated from $T_{\mathrm{bp}}(x=0)$ to $T_{1}=T_{\mathrm{bp}}\left(x_{1}\right)$, the boiling point of the solution $S_{1}$.

2. An amount of solvent equal to $m$ moles is evaporated from the solution $S_{1}$ and constitutes the vapor $W_{0}$; the concentration of the solution increases up to $x_{0}$ and the temperature increases from $T_{1}$ to $T_{\mathrm{bp}}\left(x_{0}\right)$.

3. Both the evaporated solvent and the remaining solution $S_{0}$ are cooled from $T_{\mathrm{bp}}\left(x_{0}\right)$ to $T_{\mathrm{bp}}(x=0)$.

4. The solvent vapor is condensed at temperature $T_{\mathrm{bp}}(x=0)$, thus obtaining an amount $m$ of pure solvent $W_{1}$. The remaining solution $S_{0}$ is constituted by a total of $M$ moles.

In order to calculate the efficiency of the process, we define $Q_{1}, \ldots$, $Q_{4}$ as the heat absorbed by the cell in phases $1, \ldots, 4$; as usual, we attribute a positive sign to the heat absorbed by the system. We calculate them from the thermo-physical parameters (see below for an explanation of the term $Q_{2}$ ):

Fig. 3. Scheme of a multiple effect distiller. 
$Q_{1}=C\left(x_{1}\right)(M+m)\left[T_{1}-T_{\mathrm{bp}}(x=0)\right]$

$Q_{2}=\int_{\mathrm{x}_{1}}^{x_{0}}\left\{\frac{\lambda(x)}{x}+\left[C(x)+C_{\mathrm{s}}\left(\frac{x}{x_{0}} \frac{M+m}{M}-1\right)\right] \frac{\partial T_{\mathrm{bp}}(x)}{\partial x}\right\} M \frac{x_{0}}{x} \mathrm{~d} x$

$Q_{3}=-\left[C\left(x_{0}\right) M+C_{\mathrm{s}} m\right]\left[T_{\mathrm{bp}}\left(x_{0}\right)-T_{\mathrm{bp}}(x=0)\right]$

$Q_{4}=-\lambda(0) m$,

where $C(x)$ is the molar specific heat at constant pressure of the solution at molar fraction $x, C_{\mathrm{s}}$ is the molar specific heat at constant pressure of the solvent vapor (steam), $\lambda(x)$ is the molar latent heat of evaporation of the solution at molar fraction $x$ at its boiling point. In the calculations that we show, we neglect the dependence of the specific heats on temperature. We calculate the latent heat of vaporization $\lambda(x)$ by using the Clausius-Clapeyron relation, at the temperature of the system, i.e. at $T_{\mathrm{bp}}(x)$ in phase 2 and $T_{\mathrm{bp}}(0)$ in phase 4.

The expression of $Q_{2}$ in Eq. (21) is not straightforward and deserves a discussion. The integration variable represents the concentration of the solution, which ranges between $x_{1}$ and $x_{0}$. The two addends enclosed in the curly brackets represent the latent heat adsorbed by the evaporation and the heat required for increasing the temperature of the system respectively. In turn, in the second addend, the two addends in the square brackets represent the heat capacity of the solution and of the vapor respectively.

Then we define $\Delta S_{1}, \ldots, \Delta S_{4}$ as the entropy changes underwent by the cell in phases $1, \ldots, 4$; we calculate them from the thermo-physical parameters:

$\Delta S_{1}=C\left(x_{1}\right)(M+m) \ln \frac{T_{1}}{T_{\mathrm{bp}}(x=0)}$

$\Delta S_{2}=\int_{\mathrm{x}_{1}}^{x_{0}}\left\{\frac{\lambda(x)}{x}+\left[C(x)+C_{\mathrm{s}}\left(\frac{x}{x_{0}} \frac{M+m}{M}-1\right)\right] \frac{\partial T_{\mathrm{bp}}(x)}{\partial x}\right\} M \frac{x_{0}}{x T_{\mathrm{bp}}(x)}$

$\mathrm{d} x$

$\Delta S_{3}=-\left[C\left(x_{0}\right) M+C_{\mathrm{s}} m\right] \ln \frac{T_{\mathrm{bp}}\left(x_{0}\right)}{T_{\mathrm{bp}}(x=0)}$

$\Delta S_{4}=-\frac{\lambda(0) m}{T_{\mathrm{bp}}(x=0)}$.

We calculate $\Delta G$ as:

$\Delta G=Q_{1}+Q_{2}+Q_{3}+Q_{4}-T_{\mathrm{bp}}(x=0)\left(\Delta S_{1}+\Delta S_{2}+\Delta S_{3}+\Delta S_{4}\right)$,

which relies on the hypothesis that the whole cycle is isobaric, so that no mechanical work is produced.

The heat flow from the hot source is $Q_{\mathrm{H}}=Q_{1}+Q_{2}$, thus the energy efficiency is:

$\eta=\frac{\Delta G}{Q_{\mathrm{H}}}=\frac{\Delta G}{Q_{1}+Q_{2}}$.

In order to increase the efficiency, the heat $Q_{3}$, released in step 3, can be used for heating during steps 1 and 2, by means of a countercurrent heat exchanger. We will refer to this option as the "heat recovery", where $Q_{\mathrm{H}}<Q_{1}+Q_{2}$. The analysis for distillation with heat recovery will be presented later.

\subsection{Approximated evaluation of the efficiency}

We get a very rough approximation of the efficiency $\eta$ by neglecting the heat capacities $C$ and $C_{\mathrm{s}}$, by assuming that the molar latent heat of vaporization is a constant and that the evaporation takes place at a constant temperature. The heat exchanges take place only during steps 2 and 4:

$Q_{1} \approx 0$

$Q_{2} \approx \lambda m$
$Q_{3} \approx 0$

$Q_{4} \approx-\lambda m$

and

$S_{1} \approx 0$

$S_{2} \approx \frac{\lambda m}{T_{\mathrm{bp}}\left(x_{0}\right)}$

$S_{3} \approx 0$

$S_{4} \approx-\frac{\lambda m}{T_{\mathrm{bp}}(x=0)}$

We get:

$\Delta G \approx\left(1-\frac{T_{\mathrm{bp}}(x=0)}{T_{\mathrm{bp}}\left(x_{0}\right)}\right) \lambda m$

$Q_{\mathrm{H}} \approx \lambda m$.

The efficiency is thus:

$\eta \approx 1-\frac{T_{\mathrm{bp}}(x=0)}{T_{\mathrm{bp}}\left(x_{0}\right)}$

which corresponds to the equality in the single-effect Carnot limit, Eq. (18).

The approximation of a constant temperature during evaporation holds if the variation of concentration is negligible; this is obtained in the limit of a negligible amount of solvent evaporated from the solution. This limit can be expressed in terms of the "recovery rate" $Y=m /(m$ $+M$ ), representing the ratio between the moles $m$ of distilled water $W_{0}$ and the moles $m+M$ of feed solution $S_{1}$. The limit of a negligible amount of evaporated solvent corresponds to $Y \rightarrow 0$. However, under this limit, the heat needed for taking the solution at $T_{\mathrm{bp}}\left(x_{0}\right)$ is no more negligible with respect to the (vanishing) latent heat of evaporation. In the following sections we will make a more realistic evaluation of the efficiency.

\subsection{Batch process without heat recovery}

The value of $\eta_{\text {single-effect }}$, calculated with values of the parameters for sodium chloride solutions in water, is shown in Fig. 5 (green line) as a function of the recovery rate $Y$. The figure also shows the single-effect Carnot limit $\eta_{\text {single-effect }}^{\max }$, evaluated from Eq. (18); it depends on $Y$ because the boiling point of the concentrated solution depends on its concentration, which, in turn, depends on $Y$. We notice that values of the order of the $60-90 \%$ of $\epsilon_{\text {single-effect }}$ are reached. Real MED work usually with $Y$ in the range of $0.3-0.5$; in this range we evaluate a relative efficiency $\epsilon_{\text {single-effect }}$ around $80 \%$.

We see that the efficiency $\eta_{\text {single-effect }}$ increases, when $Y$ increases; this is consistent with the higher temperature difference exploited by the process: $x_{0}$ increases with $Y$, in turn leading to an increase of the boiling point of the concentrated solution. However, in step 2, the temperature must rise from $T_{\mathrm{bp}}\left(x_{1}\right)$ to $T_{\mathrm{bp}}\left(x_{0}\right)$; the entropy production in this step becomes larger for larger $x_{0}$, and in turn for larger $Y$, leading to a decrease of the relative efficiency $\epsilon_{\text {single-effect }}$.

In the other limit, $Y \rightarrow 0$, we observe a decrease of both the efficiency $\eta_{\text {single-effect }}$ and of the relative efficiency $\epsilon_{\text {single-effect }}$, due to the increase of the heat wasted for heating the solution from $T_{\mathrm{bp}}(x=0)$ up to $T_{\mathrm{bp}}\left(x_{0}\right)$. The heat recovery described in Section 4.5 mitigates this effect.

The actual efficiency of a practical thermal distillation process is significantly higher than the results shown in Fig. 5, because multiple effects, that reuses the discharged heat several times, are usually employed. 


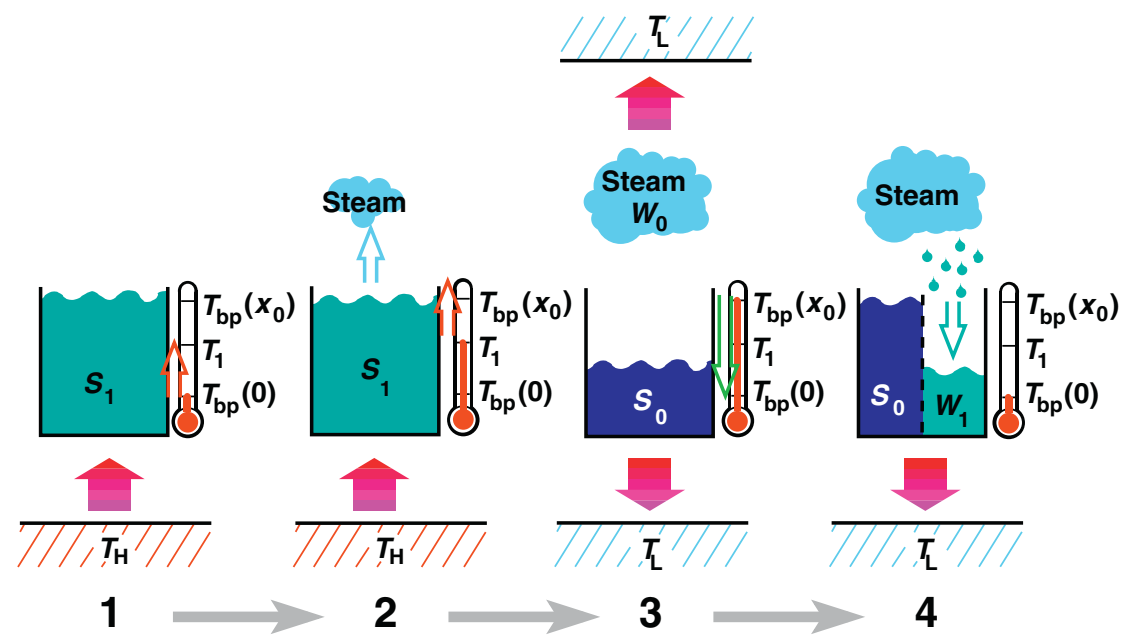

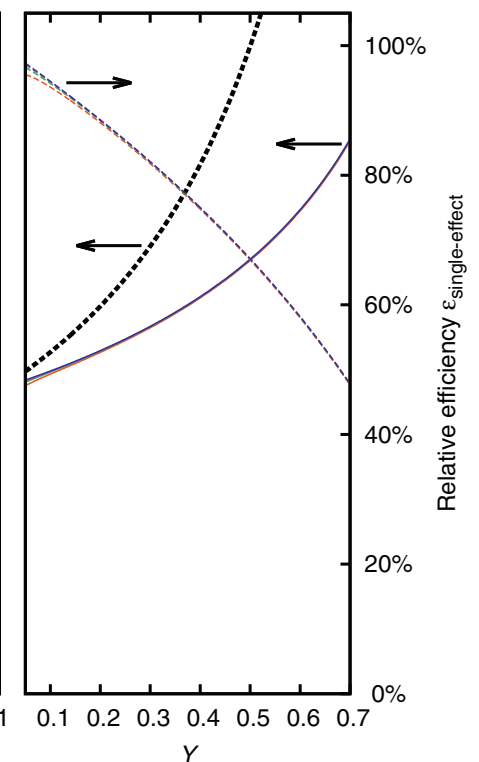

Fig. 4. Batch distillation process.
Fig. 5. Efficiency of a single effect as a function of the recovery rate $Y$. The efficiency is represented by solid lines and refers to the axis on the left; the relative efficiency is represented by dotted lines and refers to the axis on the right. The solution is $\mathrm{NaCl}$ in water; the molar fraction of the feed solution is $x_{1}=0.01$, approximately corresponding to sea water. The pressure is $1 \mathrm{~atm}$; the steam is thus condensed at $100{ }^{\circ} \mathrm{C}$. The dotted vertical line represents the saturation limit of $\mathrm{NaCl}$ solutions. The panel on the right shows the working range of actual MEDs (0.3-0.5). The various curves have been obtained with different values of temperature difference $\Delta T_{\text {he }}$ across the counter-current heat exchangers. (For interpretation of the references to color in this figure legend, the reader is referred to the web version of this article.)

\subsection{Batch process with heat recovery}

Heat is released in steps 3 and 4; here we consider the possibility of recovering it as a contribution to heat required in steps 1 and 2 .

The heat is released in step 4 at a temperature that is lower than the temperature of the system in steps 1 and 2, thus $Q_{4}$ cannot be recovered in any way. In order to evaluate the feasibility of the recovery of the heat released in step 3, it is necessary to compare the released and adsorbed heat and the temperatures at which the heat is exchanged.

The released heat per unit temperature variation is smaller during step 3 than the heat adsorbed per unit temperature variation during steps 1 and 2. Indeed, comparing steps 1 and 3, we see that the heat capacity is smaller because part of the total $M+m$ moles are composed by vapor, which has a heat capacity smaller than the condensed phase:

$\left.\frac{\partial Q}{\partial T}\right|_{3}=C\left(x_{0}\right) M+C_{\mathrm{s}} m<C\left(x_{1}\right)(M+m)=\left.\frac{\partial Q}{\partial T}\right|_{1}$
For comparing steps 2 and 3, we must consider that the increase of temperature follows from the increase of concentration, which, in turn, requires the adsorption of the latent heat of vaporization:

$\left.\frac{\partial Q}{\partial T}\right|_{3}<\left\{\frac{\lambda(x)}{x}+\left[C(x)+C_{\mathrm{s}}\left(\frac{x_{0}}{x} \frac{M+m}{M}-1\right)\right]\right\} M \frac{x_{0}}{x}=\left.\frac{\partial Q}{\partial T}\right|_{2}$

We checked the feasibility of the above-described heat exchange in the counter-current heat exchanger, by plotting the temperatures in steps 1, 2 and 3 . We see that it is actually possible, at least in principle, to perform the cooling in step 3 by sending the heat to the solutions undergoing heating in steps 1 and 2; conversely, heat from the heat source will be needed for completing the heating in steps 1 and 2 . We assume that a fixed temperature difference $\Delta T_{\text {he }}$ is present across the counter-current heat exchanger, which is constant across the whole process. The heat adsorbed from the heat source, after detraction of the heat recovered from step 3 , is thus:

$Q_{\mathrm{H}}=Q_{1}+Q_{2}-\left[C\left(x_{0}\right) M+C_{\mathrm{s}} m\right]\left[T_{\mathrm{bp}}\left(x_{0}\right)-T_{\mathrm{bp}}(x=0)-\Delta T_{\mathrm{he}}\right]$ 
The resulting efficiency is shown in Fig. 5 for various values of $\Delta T_{\text {he }}$. We notice that the values of $\Delta T_{\text {he }}$ are unrealistically small; for realistic values, of the order of a few $\mathrm{K}$ at least, no improvement can be seen with respect to the absence of heat recovery. The reason is that the available temperature difference driving the heat from step 3 to steps 1 and 2 is less than the boiling point elevation $(\approx 0.5 \mathrm{~K})$, thus the temperature drop $\Delta T_{\text {he }}$ should be much less than $0.5 \mathrm{~K}$ for having an impact on the process.

We notice that the heat recovery significantly increases the efficiency only for small values of recovery rate $Y$, i.e. for a very small amount of liquid evaporated with respect to the feed solution. Indeed, this is the limit in which the heat needed to take the feed solution to the boiling point is relevant with respect to the latent heat of vaporization.

In Fig. 5 we also report $\eta_{\text {single-effect }}^{\max }$, evaluated according to Eq. (18). We notice that the curve for ideal heat recovery, $\Delta T_{\text {he }}=0$, approximately approaches the single-effect Carnot limit efficiency $\eta_{\text {single-effect }}^{\text {max }}$ in the limit of vanishing $Y \rightarrow 0$. However, an analytical calculation shows that the limit is smaller than $\eta_{\text {single-effect }}^{\max }$; the difference is small in the case of the feed solution that we consider (similar to sea water) but can be much more relevant, e.g. in hypersaline brine and in concentrated solutions of hydroxides. It is thus important to emphasize that the distillation process can not approach the thermodynamic reversibility, i.e., entropy production is inevitable in thermal distillation even with hypothetically perfect heat recovery.

As already mentioned, the effect of the heat recovery can be only seen with unrealistically small values of $\Delta T$, the temperature drop across the heat exchangers, at least for the solutions relevant in the field of desalination. Moreover, the values of $Y$ at which the effect can be seen are much smaller than the actual values of for real processes, which usually work at in the range between 0.3 and 0.5 . We can thus conclude that the heat recovery does not have impact in the practical working conditions of a single-stage batch process. For this reason, in the following we will not consider heat recovery described in this section.

\section{Temperature drop across the heat exchangers and multiple- effect distillation}

In the discussion of the single effect, we have considered the temperatures of the solutions, i.e. we have not taken into account the temperature drop across the heat exchangers. In this section we discuss the effect of the temperature drop across the heat exchangers. Moreover, we will consider the association of effects, i.e. the so-called multiple-effect distillation; a scheme is shown in Fig. 3.

\subsection{Model of the multiple-effect distiller}

The heat flow in a multiple-effect distiller is presented in Fig. 6. The heat released during condensation (step 4) is used for the evaporation in the following stage (steps 1 and 2), so that more than one effect works with a given amount of incoming heat. The sequence of effects are at decreasing pressures $P_{\mathrm{n}}$, so that the boiling temperatures are decreasing. As already noticed, maintaining the vacuum does not require a significant mechanical power, even in practically relevant conditions.

As already discussed in Section 4.5, under the conditions that we are considering here, the heat released in step 3 is negligible and its recovery does not significantly increase the efficiency. Thus we will neglect this heat recovery.

We consider a multiple-effect distiller with $N$ effects. The condensation in the $n$th effect takes place at temperature $T_{\mathrm{bp}}\left(P_{\mathrm{n}}, x_{0}\right)$; through a heat exchanger, the latent heat of condensation is used for boiling the solution in the following effect. This leads to the following relation:

$T_{\mathrm{bp}}\left(P_{\mathrm{n}+1}, x_{0}\right)<T_{\mathrm{bp}}\left(P_{\mathrm{n}}, x=0\right)$.
In particular, we will assume that the temperature drop across the heat exchangers is a fixed value, $\Delta T_{\text {me: }}$ :

$T_{\mathrm{bp}}\left(P_{\mathrm{n}+1}, x_{0}\right)+\Delta T_{\mathrm{me}}=T_{\mathrm{bp}}\left(P_{\mathrm{n}}, x=0\right)$.

For the first and last effects:

$T_{\mathrm{H}}=T_{\mathrm{bp}}\left(P_{1}, x_{0}\right)+\Delta T_{\mathrm{me}}$

$T_{\mathrm{L}}=T_{\mathrm{bp}}\left(P_{\mathrm{N}}, x=0\right)-\Delta T_{\mathrm{me}}$.

We must also consider that the solution $S_{1}$ is at temperature $T_{\mathrm{L}}$ and it must be heated up to $T_{\mathrm{bp}}\left(P_{\mathrm{n}}, x_{1}\right)$ before entering the $n$th effect. We assume that this operation is accomplished by a counter-current heat exchange with the produced solutions $S_{0}$ and $W_{1}$, and by providing the missing amount of heat from the heat source. In order to represent the non-ideality of the counter-current heat exchanger, we will assume that a temperature drop $\Delta T_{\mathrm{i}}$ is present across them.

\subsection{Results for single- and multiple-effect distillation}

Fig. 7 shows the second-law efficiency $\eta_{2 \text { nd-law }}$ of the process, calculated with the above-described model. The results shown refer to distillation of an aqueous $\mathrm{NaCl}$ solution, with $\Delta T_{\mathrm{me}}=\Delta T_{\mathrm{i}}=5 \mathrm{~K}$ and $Y=0.4$. The lines with same $\eta$ are also reported.

The figure also reports the specific energy requirement $E$, that is defined as the energy needed to produce a unit volume of distilled water:

$E=\frac{Q_{\mathrm{H}}}{V}$,

where $V$ is the volume of distilled water. For a given value of $Y$, the volume $V$ is proportional to $\Delta G$ :

$E=g \frac{Q_{\mathrm{H}}}{\Delta G}=\frac{g}{\eta}$,

where $g$ is a constant of proportionality. In the case of Fig. 7, with $x_{0}=0.01$ and $Y=0.4$, we find $g=1.12 \mathrm{kWh} / \mathrm{m}^{3}$.

The results presented in Fig. 7 are in good agreement with empirical data of actual thermal distillation facilities using multiple stages and with heat recovery, and also with other analytical studies. The ranges of $\eta_{2 \text { nd-law }}$ and $E$ in Fig. 7 are consistent with typical MED and MSF thermal energy requirement of approximately $35-72$ and $45-97 \mathrm{kWh} / \mathrm{m}^{3}$, respectively [14-16], and second-law efficiencies of $\approx 4.2-5.9 \%$ [17-19]. The low $\eta_{2 \text { nd-law }}$ and high energy consumption of thermal distillation found in this investigation and across previous studies can be explained by application of the thermodynamic constraint described by Eq. (7), to the relatively low boiling point elevation of seawater feed to freshwater product $(\approx 0.5 \mathrm{~K})$. While incorporating additional stages can enhance $\eta_{\text {2nd-law }}$ and $E$, these improvements are limited by the practical consideration of maintaining a reasonable temperature gradient across the heat exchangers, as denoted in Fig. 7 by the leveling-off of the trend lines with increasing number of effects.

\subsection{Approximate evaluation}

The total free energy production $\Delta G$ is the sum of the free energies produced by each effect, $\Delta G_{n}$; here we neglect the heat needed for heating the solutions $S_{1}$ up to $T_{\mathrm{bp}}\left(P_{\mathrm{n}}, x_{1}\right)$, so that the heat adsorbed from the heat source, $Q_{\mathrm{H}}$, then flows through all the effects:

$\Delta G=\sum_{\mathrm{n}=1}^{\mathrm{N}} \eta_{\mathrm{n}} Q_{\mathrm{H}}$

where $\eta_{\mathrm{n}}$ is the efficiency of the $n$-th effect.

We write the overall efficiency and express the efficiencies of the $n$ th effect in terms of the relative efficiency $\epsilon_{\text {single-effect }}{ }^{\text {}}$ : 




Fig. 6. Scheme of a multiple-effect distiller.

$\eta=\frac{\Delta G}{Q_{\mathrm{H}}}=\sum_{\mathrm{n}=1}^{\mathrm{N}} \epsilon_{\text {single-effect }}^{\mathrm{n}} \frac{T_{\mathrm{bp}}\left(P_{\mathrm{n}}, x_{0}\right)-T_{\mathrm{bp}}\left(P_{\mathrm{n}}, 0\right)}{T_{\mathrm{bp}}\left(P_{\mathrm{n}}, x_{0}\right)}$.

As already noticed, according to "Dühring's rule" [35] (see Eq. (19)), we can assume that the terms of the summation do not depend on the pressure; we thus decide to evaluate all the boiling temperatures at pressure $P_{1}$. For the same reason, we assume that $\epsilon_{\text {single-effect }}$ is a constant (from the analysis above, we know that it is of the order of $80 \%$ in practical cases):

$\eta=N \epsilon_{\text {single-effect }} \frac{T_{\mathrm{bp}}\left(P_{1}, x_{0}\right)-T_{\mathrm{bp}}\left(P_{1}, 0\right)}{T_{\mathrm{bp}}\left(P_{1}, x_{0}\right)}$.

We rewrite this last equation by defining $\Delta T_{\mathrm{bp}}$ the boiling point elevation $T_{\mathrm{bp}}\left(P_{1}, x_{0}\right)-T_{\mathrm{bp}}\left(P_{1}, 0\right)$, which is roughly equal for all the effects, and by expressing $T_{\mathrm{bp}}\left(P_{1}, x_{0}\right)$ as a function of $T_{\mathrm{H}}$ : $\eta \approx N \epsilon_{\text {single-effect }} \frac{\Delta T_{\mathrm{bp}}}{T_{\mathrm{H}}-\Delta T_{\mathrm{me}}}$.

The temperature drop across each effect is approximately $\Delta T_{\mathrm{bp}}+\Delta T_{\mathrm{me}}$. The sum of all the temperature drops is $T_{\mathrm{H}}-T_{\mathrm{L}}-\Delta T_{\mathrm{me}}$ : $N\left(\Delta T_{\mathrm{bp}}+\Delta T_{\mathrm{me}}\right)=T_{\mathrm{H}}-T_{\mathrm{L}}-\Delta T_{\mathrm{me}}$.

By substituting the value of $N$ extracted from this equation, we get: $\eta \approx \epsilon_{\text {single-effect }} \frac{T_{\mathrm{H}}-T_{\mathrm{L}}-\Delta T_{\mathrm{me}}}{\Delta T_{\mathrm{bp}}+\Delta T_{\mathrm{me}}} \frac{\Delta T_{\mathrm{bp}}}{T_{\mathrm{H}}-\Delta T_{\mathrm{me}}}$.

We calculate the second-law efficiency $\eta_{2 \text { nd-law }}$ according to Eq. (13):

$\eta_{\text {2nd-law }} \approx \epsilon_{\text {single-effect }} \frac{T_{\mathrm{H}}-T_{\mathrm{L}}-\Delta T_{\mathrm{me}}}{\Delta T_{\mathrm{bp}}+\Delta T_{\mathrm{me}}} \frac{\Delta T_{\mathrm{bp}}}{T_{\mathrm{H}}-\Delta T_{\mathrm{me}}} \frac{T_{\mathrm{H}}}{T_{\mathrm{H}}-T_{\mathrm{L}}}$.

This rough evaluation is shown in Fig. 7 as a solid black line, with $\epsilon_{\text {single-effect }}$ evaluated for the effect at higher temperature. We see that Eq. (51) is a good approximation; it is excellent when a perfect heat exchange $\Delta T_{\mathrm{i}}=0 \mathrm{~K}$ is considered.

We can further approximate Eq. (51) by taking $\epsilon_{\text {single-effect }}=1$ and $T_{\mathrm{H}}-\Delta T_{\mathrm{me}} \approx T_{\mathrm{H}}$ :

$\eta_{\text {2nd-law }} \approx \frac{\Delta T_{\mathrm{bp}}}{\Delta T_{\mathrm{bp}}+\Delta T_{\mathrm{me}}}$.

This value represents an upper limit of the second-law efficiency, determined by the ratio between the boiling point elevation and the temperature drop across the heat exchangers. The 2nd-law efficiency $\eta_{\text {2nd-law }}$ can only approach $100 \%$ when $\Delta T_{\text {me }} \ll \Delta T_{\text {bp }}$, a condition that cannot be practically met in real plants. This is the reason for the low second-law efficiency of distillation of sea water. Using a multiple-effect distiller allows to approach the limit, as the available temperature difference $T_{\mathrm{H}}-T_{\mathrm{L}}$ increases. Our results can be compared with the analysis of the effect of the boiling point elevation on the multipleeffect distillation [21].

We also see that the second-law efficiency increases with increasing boiling point elevation, at variance with the usual belief that the boiling point elevation is detrimental for distillation efficiency. A similar result, i.e. the increase of second-law efficiency with increasing concentration, was previously reported [23].

\section{Representation in the T-S plane}

In the batch process described in Section 4.2, the system is composed by two parts, the solution and the steam, that are at the same temperature; thus the state of the system as a whole can be represented in the $T$ versus $S$ plane, as shown in Fig. 8.

It can be shown that $\Delta G$ equals the area enclosed by the path in the $T$ versus $S$ plane, after closing it connecting the end of step 4 to the beginning of step 1 . The efficiency of the Carnot cycle is, also in this case, the rectangle from $T_{\mathrm{H}}$ to $T_{\mathrm{L}}$, represented in yellow in Fig. 8 .

The presence of a temperature difference between the consecutive cycles is due to the temperature drop $\Delta T_{\text {me }}$ across the heat exchangers. It is clear that the area covered by the distillation cycle (in red) is proportional to the boiling point elevation $\Delta T_{\mathrm{bp}}$, while the part of the Carnot-like cycle is proportional to $\Delta T_{\text {me }}$, if the number of effects is enough to cover the temperature range from $T_{\mathrm{L}}$ to $T_{\mathrm{H}}$.

\section{Comparison with reverse osmosis and electrodialysis}

In this section we compare various desalination technologies based on the efficiency. A similar comparison has been reported in Ref. [37]. 
(a)

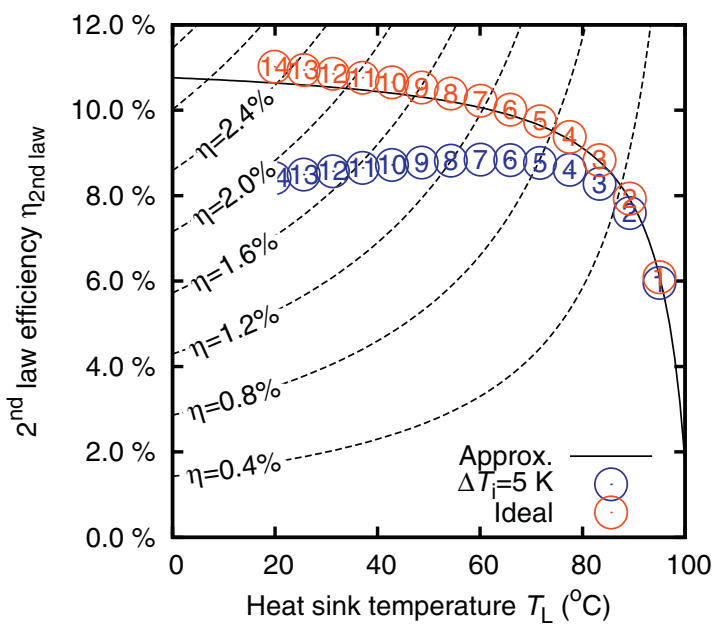

(b)

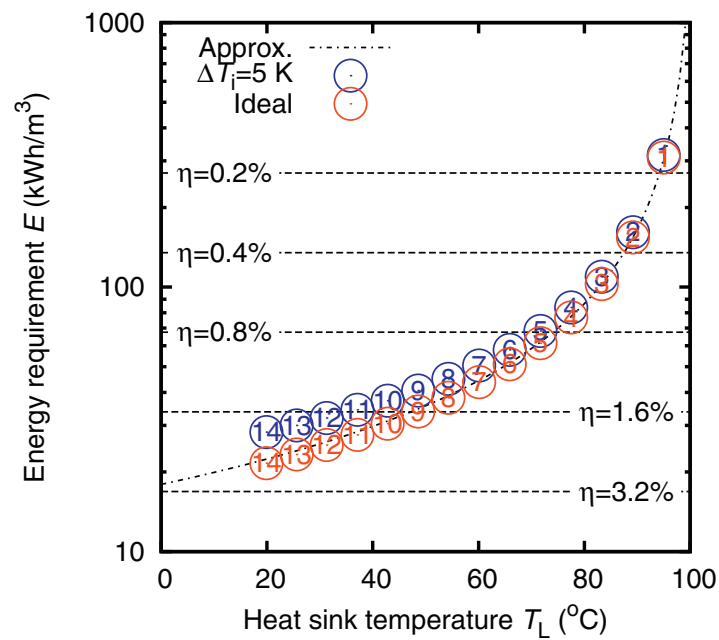

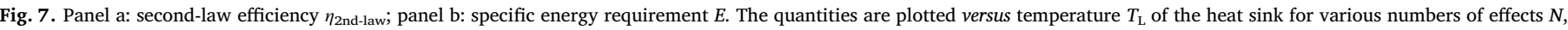

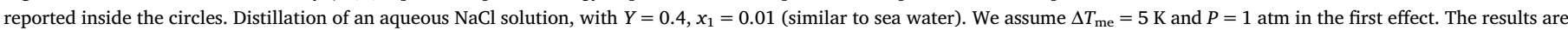

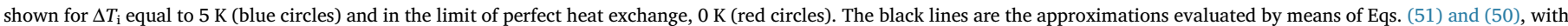

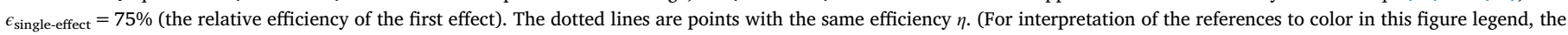
reader is referred to the web version of this article.)

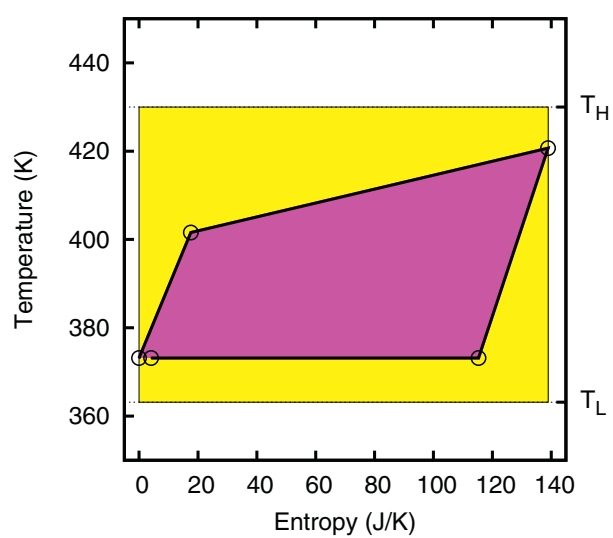

Reverse osmosis and electrodialysis are the main desalination technologies competing with thermal distillation. Analyses indicate that the membrane-based processes have second-law efficiencies in the region of $\approx 30-80 \%$ (depending primarily on feedwater salinity, recovery rate, and water/ion permeation rate) [11-13], significantly higher than the thermal distillation efficiency of $\approx 4-5 \%$ (Fig. 7a). Reverse osmosis and electrodialysis are able to achieve starkly greater $\eta_{2 \text { nd-law }}$ because the processes are not thermally-driven and are, hence, not restricted by the Carnot-like efficiency of $\eta=1-T_{\mathrm{L}} / T_{\mathrm{H}}$, Eq. (7), that MED and MSF are thermodynamically bounded by.

Electrical energy is the main power input for both the membranebased technologies. Presently, electricity is, for the most part, generated thermally in thermoelectric power plants by combusting fossil fuels to drive turbines. Therefore, thermal desalination and electrically-powered reverse osmosis and electrodialysis effectively utilize the same energy source - heat. However, because the net efficiency of thermoelectric plants is around 25-40\% [38], it is overall more efficient to convert thermal energy to electricity which is then used to power reverse osmosis or electrodialysis desalination, than it is to directly utilize the heat to drive thermal distillation.

Membrane distillation is an emergent technology that utilizes lowtemperature heat to drive the permeation of volatile compounds across a hydrophobic microporous membrane $[39,40]$. The technology can be employed for desalination, where the volatized species for separation is water. While membrane distillation has the advantage of utilizing relatively low temperatures to drive desalination, it is ultimately still a thermally-driven process. Although this study did not explicitly examine the energy requirement of membrane distillation for desalination, the process will nevertheless be confined by the thermodynamic principles and governing equations presented in this analysis, as 
supported by recent energy analysis studies [41-43].

\section{Conclusion}

Improving energy efficiency and reducing the specific energy requirement is vital to advancing the sustainability of desalination. In this study, we show that the energy efficiencies of thermal desalination, i.e., MED and MSF, are limited by physical laws.

First of all, the efficiency of any thermal separation process is limited by a law similar to the Carnot law for the heat engines. This Carnotlike efficiency dictates the fraction of heat energy, based on the high and low temperatures, that can be utilized for separation work. Therefore, for thermal desalination, the amount of water that can be produced with a given amount of heat is thermodynamically constrained, even with an ideal process.

We have shown that the efficiency of a single-effect distiller is limited by the Carnot-like law, and is further constrained by the boiling points of the solutions. This leads to a highly critical but somewhat counterintuitive insight: in distillation technology, a large boiling point elevation is usually seen as detrimental, because it seemingly imposes to increase the temperature of the heat source; however, we see that it favorably allows us to reach a higher efficiency.

The energy efficiency advantage of a high boiling point elevation persists also when various effects are associated in a multiple-effect device. In this case, another quantity becomes relevant: the temperature drop across the heat exchangers. The efficiency of the multipleeffect device is limited by the ratio between the boiling point elevation and the temperature drop across the heat exchangers.

The physical limitations discussed in this study are actually approached in real plants; as we have shown, the findings of this analysis provide the thermodynamic rationale that explains the different performances of the various technologies. Our results can be seen both as rigorous limits and as rules-of-thumb for designing and evaluating the performances of a multiple-effect distillation unit, based only on the properties of the solution to be distilled (the boiling point elevation) and on a parameter of the device (the temperature drop across the heat exchangers).

\section{References}

[1] J.J. Bogardi, D. Dudgeon, R. Lawford, E. Flinkerbusch, A. Meyn, C. Pahl-Wostl, K. Vielhauer, C. Vörösmarty, Water security for a planet under pressure: interconnected challenges of a changing world call for sustainable solutions, Curr. Opin. Env. Sust. 4 (2012) 35-43, http://dx.doi.org/10.1016/j.cosust.2011.12.002.

[2] J. Rockstrom, W. Steffen, K. Noone, Å. Persson, F. Stuart Chapin, E.F. Lambin, T.M. Lenton, A safe operating space for humanity, Nature 461 (2009) 472-475, http://dx.doi.org/10.1038/461472a.

[3] S.W. Running, A measurable planetary boundary for the biosphere, Science 337 (2012) 1458-1459, http://dx.doi.org/10.1126/science.1227620.

[4] C.J. Vorosmarty, C.J. Vörösmarty, P.B. McIntyre, M.O. Gessner, D. Dudgeon, A. Prusevich, P. Green, S. Glidden, S.E. Bunn, C.A. Sullivan, C. Reidy Liermann, P.M. Davies, Global threats to human water security and river biodiversity, Nature 467 (2010) 555-561, http://dx.doi.org/10.1038/nature09440.

[5] The United Nations world water development report 2014: water and energy, United Nations, Paris, 2014.

[6] M. Elimelech, W.A. Phillip, The future of seawater desalination: energy, technology, and the environment, Science 333 (2011) 712-717, http://dx.doi.org/10.1126 science. 1200488.

[7] N. Ghaffour, T.M. Missimer, G.L. Amy, Technical review and evaluation of the economics of water desalination: current and future challenges for better water supply sustainability, Desalination 309 (2013) 197-207, http://dx.doi.org/10. 1016/j.desal.2012.10.015.

[8] I.C. Karagiannis, P.G. Soldatos, Water desalination cost literature: review and assessment, Desalination 223 (2008) 448-456, http://dx.doi.org/10.1016/j.desal. 2007.02.071.

[9] H. Cooley, et al., Desalination: With a Grain of Salt, Pacific Institute, 2006.

[10] J.E. Miller, Review of Water Resources and Desalination Technologies, (2003).

[11] K.M. Chehayeb, J.H. Lienhard, Entropy generation analysis of electrodialysis,
Desalination 413 (2017) 184-198, http://dx.doi.org/10.1016/j.desal.2017.03.001.

12] S. Lin, M. Elimelech, Staged reverse osmosis operation: configurations, energy efficiency, and application potential, Desalination 366 (2015) 9-14, http://dx.doi. org/10.1016/j.desal.2015.02.043.

[13] C. Liu, K. Rainwater, L. Song, Energy analysis and efficiency assessment of reverse osmosis desalination process, Desalination 276 (2011) 352-358, http://dx.doi.org/ 10.1016/j.desal.2011.03.074.

[14] Y. Ghalavand, M.S. Hatamipour, A. Rahimi, A review on energy consumption of desalination processes, Desalin. Water Treat. 54 (2015) 1526-1541, http://dx.doi. org/10.1080/19443994.2014.892837.

[15] V.G. Gude, Energy storage for desalination processes powered by renewable energy and waste heat sources, Appl. Energ. 137 (2015) 877-898, http://dx.doi.org/10. 1016/j.apenergy.2014.06.061.

[16] N.M. Wade, Distillation plant development and cost update, Desalination 136 (2001) 3-12, http://dx.doi.org/10.1016/S0011-9164(01)00159-X.

[17] O.A. Hamed, M.A.K. Al-Sofi, M. Imam, G.M. Mustafa, K.B. Mardouf, H. Al-Washmi, Thermal performance of multi-stage flash distillation plants in Saudi Arabia, Desalination 128 (2000) 281-292, http://dx.doi.org/10.1016/S0011-9164(00) 00043-6.

[18] N. Kahraman, Y.A. Cengel, Exergy analysis of a MSF distillation plant, Energ. Convers. Manage. 46 (2005) 2625-2636, http://dx.doi.org/10.1016/j.enconman. 2004.11.009.

[19] K.H. Mistry, R.K. McGovern, G.P. Thiel, E.K. Summers, S.M. Zubair, J.H. Lienhard V, Entropy generation analysis of desalination technologies, Entropy 13 (10) (2011) 1829-1864, http://dx.doi.org/10.3390/e13101829.

[20] K. Mistry, J.H. Lienhard V, Generalized least energy of separation for desalination and other chemical separation processes, Entropy 15 (6) (2013) 2046-2080, http:// dx.doi.org/10.3390/e15062046.

[21] K. Mistry, M.A. Antar, J.H. Lienhard V, An improved model for multiple effect distillation, Desalination and Water Treatment 51 (2013) 807-821, http://dx.doi. org/10.1080/19443994.2012.703383.

[22] J.H. Lienhard V, K.H. Mistry, M.H. Sharqawy, G.P. Thiel, Thermodynamics, exergy, and energy efficiency in desalination systems, in: H.A. Arafat (Ed.), Desalination Sustainability: A Technical, Socioeconomic, and Environmental Approach, Elsevier Publishing Co., 2017, pp. 1-85 Ch. 4.

[23] G.P. Thiel, E.W. Tow, L.D. Banchik, H.W. Chung, J.H. Lienhard V, Energy consumption in desalinating produced water from shale oil and gas extraction, Desalination 366 (2015) 94-112, http://dx.doi.org/10.1016/j.desal.2014.12.038.

[24] M. Blahušiak, A.A. Kiss, S.R.A. Kersten, B. Schuur, Quick assessment of binary distillation efficiency using a heat engine perspective, Energy 116 (2016) 20-31, http://dx.doi.org/10.1016/j.energy.2016.09.097.

[25] J.B. Opfell, Free energy explanation of separative work, AIChE J. 24 (1978) 726-728, http://dx.doi.org/10.1002/aic.690240423.

[26] J.N. Weinstein, F.B. Leitz, Electric power from differences in salinity: the dyalitic battery, Science 191 (1976) 557-559.

[27] J.W. Post, H.V.M. Hamelers, C.J.N. Buisman, Energy recovery from controlled mixing salt and fresh water with a reverse electrodialysis system, Env. Sci. Techn 42 (2008) 5785-5790, http://dx.doi.org/10.1021/es8004317.

[28] O. Levenspiel, N. de Vevers, The osmotic pump, Science 183 (1974) 157-160.

[29] S. Loeb, Osmotic power plant, Science 189 (1975) 654-655.

[30] T.S. Chung, X. Li, R.C. Ong, Q.G.e.H. Wang, G. Han, Emerging forward osmosis (FO) technologies and challenges ahead for clean water and clean energy applications, Curr. Opin. Chem. Eng. 1 (3) (2012) 246-257.

[31] N.Y. Yip, D.A. Vermaas, K. Nijmeijer, M. Elimelech, Thermodynamic, energy efficiency, and power density analysis of reverse electrodialysis power generation with natural salinity gradients, Environ. Sci. Technol. 48 (9) (2014) 4925-4936, http:// dx.doi.org/10.1021/es5005413.

[32] N.Y. Yip, M. Elimelech, Thermodynamic and energy efficiency analysis of power generation from natural salinity gradients by pressure retarded osmosis, Environ. Sci. Technol. 46 (9) (2012) 5230-5239, http://dx.doi.org/10.1021/es300060m.

[33] K.V. Reddy, N. Ghaffour, Overview of the cost of desalinated water and costing methodologies, Desalination 205 (2007) 340-353.

[34] N.M. Wade, Technical and economic evaluation of distillation and reverse osmosis desalination processes, Desalination 93 (1993) 343-363.

[35] A. Mclaren White, A derivation of Dühring's rule, Ind. Eng. Chem. 22 (3) (1930) 230-232.

[36] A. Carati, M. Marino, D. Brogioli, Thermodynamic study of a distiller-electrochemical cell system for energy production from low temperature heat sources, Energy 93 (1) (2015) 984-993, http://dx.doi.org/10.1016/j.energy.2015.09.108.

[37] E.W. Tow, R.K. McGovern, J.H. Lienhard V, Raising forward osmosis brine concentration efficiency through flow rate optimization, Desalination 366 (2015) 71-79, http://dx.doi.org/10.1016/j.desal.2014.10.034.

[38] S.C. Kaushika, V.S. Reddy, S.K. Tyagi, Energy and exergy analyses of thermal power plants: a review, Renew. Sust. Energ. Rev. 15 (2011) 1857-1872, http://dx.doi.org/ 10.1016/j.rser.2010.12.007.

[39] E. Drioli, A. Ali, F. Macedonio, Membrane distillation: recent developments and perspectives, Desalination 356 (2015) 56-84, http://dx.doi.org/10.1016/j.desal. 2014.10.028.

[40] P. Wang, T.-S. Chung, Recent advances in membrane distillation processes: membrane development, configuration design and application exploring, J. Membr. Sci. 474 (2015) 39-56, http://dx.doi.org/10.1016/j.memsci.2014.09.016. 
[41] S. Lin, N.Y. Yip, M. Elimelech, Direct contact membrane distillation with heat recovery: thermodynamic insights from module scale modeling, J. Membr. Sci. 453 (2014) 498-515, http://dx.doi.org/10.1016/j.memsci.2013.11.016.

[42] E.K. Summers, H.A. Arafat, J.H. Lienhard, Energy efficiency comparison of singlestage membrane distillation (MD) desalination cycles in different configurations,
Desalination 290 (2012) 54-66, http://dx.doi.org/10.1016/j.desal.2012.01.004. [43] G. Zuo, R. Wang, R. Field, A.G. Fane, Energy efficiency evaluation and economic analyses of direct contact membrane distillation system using Aspen Plus, Desalination 283 (2011) 237-244, http://dx.doi.org/10.1016/j.desal.2011.04.048. 